\title{
Editorial: Promoting Inclusive and Respectful Communications
}

"Diversity, Inclusion, and Respect" is one of the explicitly stated APS values in the 2019 Strategic Plan [1]. These principles also extend to the Physical Review journals, their readers, authors, referees, and editors.

Using respectful, nonjudgmental language is essential for the exchange of ideas among scientists. Exaggerations, hyperboles, and overstatements have no place in scientific communications. They suppress the exchange of honest ideas rooted in facts and data.

This also extends to language that makes certain groups of scientists feel unwelcome. Any biases against members of the scientific community because of their race, color, ethnicity, national origin, religion, disability, sexual orientation, gender expression, or gender identity excludes them from contributing equally to the progress of science.

When we create a welcoming environment that is respectful, inclusive, and equitable, we ensure that science and all who practice it have every opportunity to be successful. When marginalized individuals and communities feel excluded and not welcome, it can reduce their productivity and career advancement.

One way of being respectful and inclusive is the correct use of chosen pronouns. When authors choose to list their email addresses in footnotes in their papers, they invite readers to contact them for further discussions, information, questions, and suggestions. In these communications it is beneficial to know which pronouns to use to address the authors.

Thus, we now give authors the opportunity to list their pronouns together with their email addresses. Adding one's pronouns (for example she/her/hers, he/him/his, they/them/theirs) indicates that one is mindful of the diversity of our authors. It also normalizes the use of pronouns to respect scientists and increases the acceptance of all gender identities in science. We note that the singular third person pronouns they/them/theirs are grammatically correct with an established history and have been recommended in the LGBT+ Climate in Physics Report [2].

The first paper where the authors have used this opportunity was recently published in Physical Review B [3].

Furthermore, although we do not have data from our journals, there are many studies reporting implicit biases within all scientific communities, and there is no reason to believe that these biases are not prevalent in physics in general and within communications during the publication process in particular. Thus, we are committed to uphold APS values to adhere to using factual and unbiased language in communications and papers.

The Physical Review journals recently signed on to the Royal Society of Chemistry initiative Joint commitment for action on inclusion and diversity in publishing [4] stating "As a group, we acknowledge that biases exist in scholarly publishing and we commit to scrutinizing our own processes to minimize these. We will pool our resources, expertise and insight to accelerate research culture change."

As a first step we are addressing the issue of gender biases in the correspondence and manuscripts. It is quite common in referee reports and even more so in authors' responses to assume that the author or referee is male when not known. It also occasionally occurs in manuscripts when referring to authors of other papers and in acknowledgments (for example, "Prof. Jones and his collaborators").

We have revised our instructions to authors, referees, and copy editors to use gender-neutral language. We follow the guidance of the latest edition of the Chicago Manual of Style [5], which lists nine techniques for achieving gender neutrality.

In conclusion, APS and the Physical Review journals strongly encourage the use of inclusive and respectful language, which includes the use of the name and pronoun of a person's choosing and using gender-neutral phrasing whenever possible. This is only the first step in our efforts, 
and we hope that it will help avoid biases and form an accepting and welcoming environment for everyone.

\author{
Michael Thoennessen \\ Editor in Chief \\ American Physical Society
}

(2) Published 18 November 2020

DOI: 10.1103/PhysRevPhysEducRes.16.020001

[1] https://www.aps.org/about/strategicplan/upload/APSStrat Plan2019.pdf.

[2] https://www.aps.org/programs/lgbt/upload/LGBTClimatein PhysicsReport.pdf.

[3] J. Lohr, A. L. Larralde, J. Curiale, R. Sánchez, J. Campo, G. J. Cuello, D. Sheptyakov, L. Keller, M. Kenzelmann, and
G. Aurelio, Intermediate magnetic phase of the magnetoelectric compound $(\mathrm{Ca}, \mathrm{Sr}) \mathrm{BaCo}_{4} \mathrm{O}_{7}$ described with the superspace formalism, Phys. Rev. B 102, 134406 (2020).

[4] https://www.rsc.org/new-perspectives/talent/jointcommitment-for-action-inclusion-and-diversity-in-publishing/.

[5] https://www.chicagomanualofstyle.org/home.html. 